\title{
Role of nitric oxide and NADPH oxidase-derived superoxide in regulating coronary blood flow and metabolism in cardio-metabolic diseases
}

\author{
Akos Koller ${ }^{1,2 *}$ \\ ${ }^{1}$ Dept. of Pathophysiology and Gerontology, Medical School, and Szentagothai Research Centre, \\ University of Pecs, Pecs, Hungary \\ ${ }^{2}$ Dept. of Physiology, New York Medical College, Valhalla, United States of America
}

In cardio-metabolic diseases both the coronary circulation and cardiac metabolism are altered. We have studied a condition called hyperhomocysteinemia (HHcy), which can develop as a result of genetic or environmental causes. This metabolic disease is underappreciated, yet even mild or moderate elevation of plasma concentrations of homocystein (Hcy, a sulfur-containing amino acid produced via methionine metabolism) leads to coronary and peripheral arterial and vascular diseases, increased thrombosis and consequently increased mortality. The underlying mechanisms have not been revealed yet.

Our recent studies indicate that there are common pathomechanisms, which may affect all of the cellular functions involved. We have shown that a dysfunction of nitric oxide (NO) mediation of dilator responses in isolated rat arterioles with methionine diet-induced hyperhomocysteinemia develops (Arterioscler Thromb Vasc Biol. 1999;19(8):1899-904) with simultaneously increased TXA2 the activity in arterioles and platelets (Arterioscler Thromb Vasc Biol. 2000;20(5): 1203-8). This was due to an oxidative stress-induced dys-

Received: $1^{\text {st }}$ May 2014

*Address for correspondence: Dept. of Pathophysiology and Gerontology, Medical School, University of Pecs, 7624 Pecs, Szigeti str. 12, Hungary.

Email: akos.koller@aok.pte.hu regulation of arteriolar wall shear stress and blood pressure (Am J Physiol Heart Circ Physiol. 2003;285(6):H2277-83). Moreover, HHcy elicited flow-induced constrictions of venules due to increased cyclooxygenase-2 derived thromboxane A2 and reactive oxygen species (Atherosclerosis. 2010;208( 1):43-9). The alteration in the endothelial function affected the ability of NO to regulate cardiac metabolism as we have found that elevated levels of p22phox, p67phox, and rac-1 indicate an increased NADPH oxidase assembly resulting in an increased superoxide production and in a reduced ability of $\mathrm{NO}$ to regulate the mitochondrial function in the myocardium (Circulation. 2005;111(16):2112-8.). The interaction of these pathomechanisms explain why HHcy increases the uptake of glucose and lactate and decreases the uptake of free fatty acid by the heart (Circulation. 2007;115(2):25562). Similar alterations could play a role in the development of cardio-metabolic and coronary dysfunction in other metabolic diseases, such as diabetes mellitus, hyperlipidemia and metabolic syndrome.

KEYWORDS: nitric oxide, homocystein, metabolic disease. CITATION: Cardiol Croat. 2014;9(5-6):255.

Support: NIH grants P01-HL-43023, R01-HL-50142 and HL-63129 to Dr. Hintze, P01-HL-74237 to Dr. Recchia, and HL-46813 and AHA 0555897T and 0855910D. Hungarian Scientific Research Fund (OTKA T-023863, T033117 and T-34779, K 108444 to Dr. Koller. 